\title{
The insulin hypersecretion hypothesis: cause or effect?
}

\author{
Constantin Polychronakos ${ }^{1}$
}

Received: 1 October 2021 / Accepted: 5 October 2021 / Published online: 18 December 2021

(C) The Author(s), under exclusive licence to Springer-Verlag GmbH Germany, part of Springer Nature 2021

\author{
Abbreviation \\ GDM Gestational diabetes mellitus
}

To the Editor: I read with interest the paper by Perng and colleagues, entitled 'How does exposure to overnutrition in utero lead to childhood adiposity? Testing the insulin hypersecretion hypothesis in the EPOCH cohort' [1]. I found it particularly revealing that offspring of obese women and/or women with gestational diabetes mellitus (GDM) have earlyonset hyperinsulinaemia, even when the effect of increased adiposity is all but eliminated by studying only offspring with normal BMI at age 10 years. However, a critical examination of the data raises serious doubts about the conclusions offered in the paper insofar as the paper's main claim in support of the primary insulin hypersecretion hypothesis is not justified by the evidence presented.

'Overnutrition in utero', as defined in the paper, is not the only factor that can account for the effect seen in the exposed offspring. Insulin resistance, a feature of GDM [2], is heritable even after adjusting for BMI [3]. Excess adiposity is the most common but not the only cause of insulin resistance and one might expect some inherited genetic load for non-obesity related insulin resistance in offspring of women with GDM, after excluding the one-third of all eligible offspring who were already overweight (the latter, by the way, explainable by the heritability of early-onset obesity [4]). Genetically inherited insulin resistance can explain the main findings in Table 1 of Perng et al [1]. Exposed offspring have increased HOMA-IR, which parallels their hyperinsulinaemia, creating a chicken/ egg question regarding the direction of causality between insulin hypersecretion and resistance. No evidence is offered in the paper to support the direction proposed. In fact, the observation that blood glucose levels are so precisely equal

Constantin Polychronakos

Constantin.Polychronakos@McGill.ca

1 The Research Institute of the McGill University Health Centre and the Montreal Children's Hospital, Montréal, Québec, Canada to control, attests to the exquisite precision with which the normal (i.e. non-hyperfunctioning) beta cell adjusts insulin secretion to compensate for insulin resistance. Hyperinsulinaemia, as the primary driver prior to and in the absence of insulin resistance, should cause hypoglycaemia or at least distinctly lower glucose levels within the normal range; these were not observed at baseline, making primary insulin resistance much more likely. Therefore, support for the insulin hypersecretion hypothesis remains, to use the authors' own words, 'limited and inconsistent'.

Funding The author's research is funded by the Canadian Institutes of Health Research and by Genome Canada.

Author's relationships and activities The author is Chief Scientific 61 Officer of MaiDa, a for-profit enterprise offering genetic testing.

Contribution statement The author was the sole contributor to this 64 paper.

\section{References}

1. Perng W, Kelsey MM, Sauder KA, Dabelea D (2021) How does exposure to overnutrition in utero lead to childhood adiposity? Testing the insulin hypersecretion hypothesis in the EPOCH cohort. Diabetologia 64:2237-2246. https://doi.org/10.1007/s00125-02105515-2

2. Ryan EA, O'Sullivan MJ, Skyler JS (1985) Insulin action during pregnancy. Studies with the euglycemic clamp technique. Diabetes 34(4):380-389. https://doi.org/10.2337/diab.34.4.380

3. Chen J, Spracklen CN, Marenne G et al (2021) The trans-ancestral genomic architecture of glycemic traits. Nat Genet 53(6):840-860. https://doi.org/10.1038/s41588-021-00852-9

4. Meyre D, Delplanque J, Chèvre JC et al (2009) Genome-wide association study for early-onset and morbid adult obesity identifies three new risk loci in European populations. Nat Genet 41(2):157-159. https://doi.org/10.1038/ng.30

Publisher's note Springer Nature remains neutral with regard to jurisdictional claims in published maps and institutional affiliations. 